\title{
Room Temperature Hydrogen Absorption of Titanium with Surface Modification by Organic Solvents
}

Keita Shinzato $^{\dagger}$, So Hamamoto ${ }^{\dagger}$, Hiroki Miyaoka ${ }^{\ddagger}$, Takayuki Ichikawa ${ }^{* \dagger}$

${ }^{\dagger}$ Graduate School of Engineering, Hiroshima University, 1-4-1 Kagamiyama, HigashiHiroshima, 739-8527, Japan

${ }^{\ddagger}$ Natural Science Center for Basic Research and Development, Hiroshima University, 1-3-1 Kagamiyama, Higashi-Hiroshima, 739-8530, Japan 


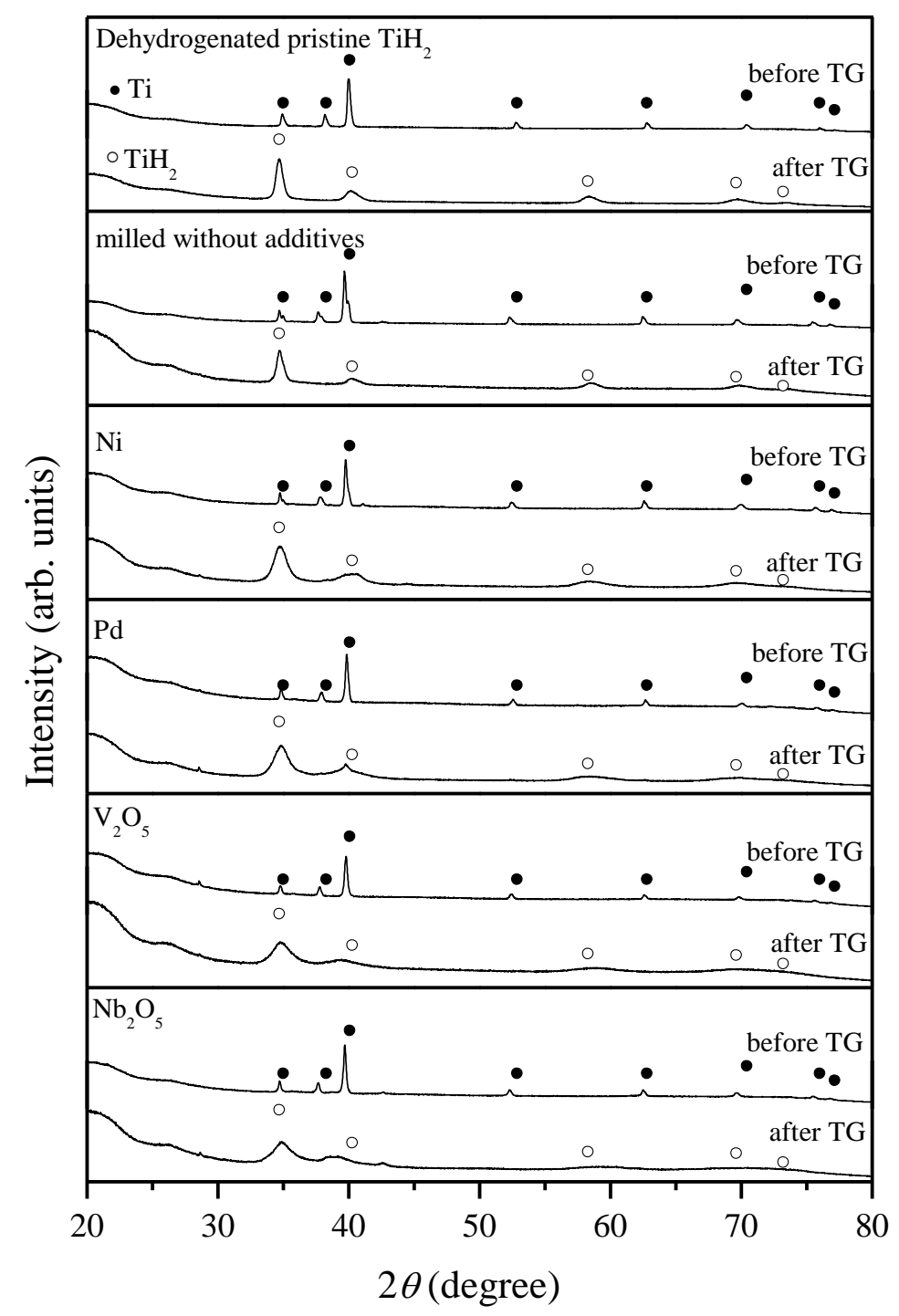

Figure S1. XRD results of the Ti milled with various kinds of additives before and after TG measurements. 


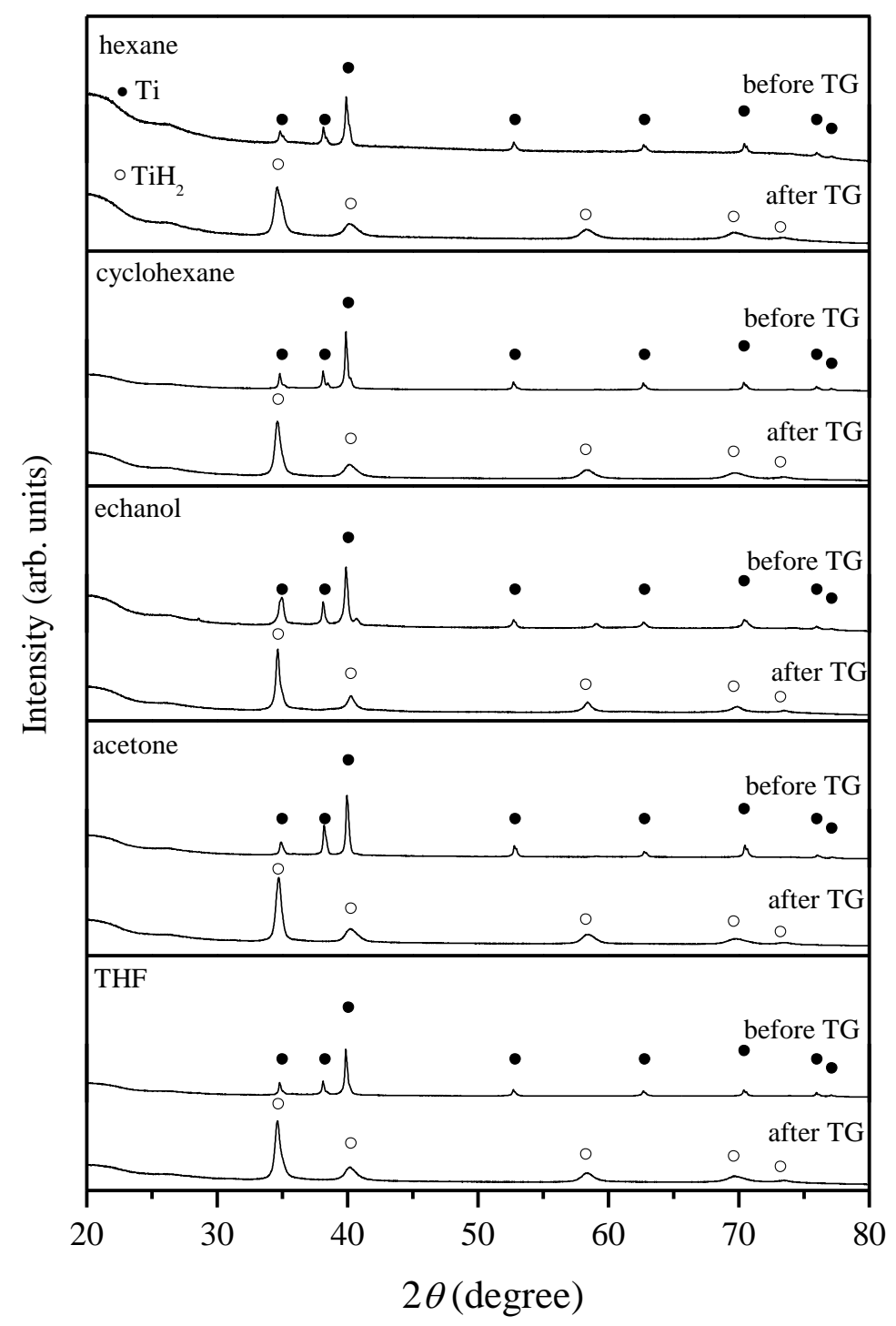

Figure S2. XRD results of the Ti milled with various kinds of organic solvents before and after TG measurements. 